\title{
Possible leap ahead in filovirus therapeutics
}

\author{
Cell Research (2014) 24:647-648. doi:10.1038/cr.2014.49; published online 15 April 2014
}

In a recent study published in $\mathrm{Na}$ ture, Warren et al. describe the generation of a novel synthetic adenosine analogue, $B C X 4430$, a synthetic druglike small molecule that provides protection from Ebola and Marburg virus infection in animal models.

While this is not the first small molecule with in vivo efficacy against filovirus infections (as summarized in [1]), it is the first showing efficacy in a nonhuman primate model. Clinically useful treatments for Ebola and Marburg viruses have been elusive and until now, potential post-exposure therapies have faced practical, regulatory, safety and/or cost obstacles. BCX4430 demonstrated up to $100 \%$ protection when administered as late as $48 \mathrm{~h}$ following infection with Marburg virus (strain Musoke) in the cynomolgus macaque model. Lower viremia, reduced clotting times and reduced liver enzyme levels were observed in treated animals. In addition, this novel nucleoside analogue showed comparable in vitro activity against other strains of Marburg virus as well as different species of Ebola virus. Antiviral activity was also observed in Marburg and Ebola virus mouse and guinea pig models. The incorporation of BCX4430 monophosphate into the nascent viral RNA chain appears to lead to chain termination. Notably, there was no evidence of incorporation of BCX4430 into human RNA or DNA [1]. In addition, broad spectrum in vitro activity, albeit at higher drug concentrations, was observed against selected bunyaviruses, paramyxoviruses, picornaviruses and flaviviruses.

Since the first description of postexposure therapeutics for filoviruses, the window of opportunity for treatment and the survival rate from these treatments in nonhuman primate experiments have been increasing (Figure 1). Experimental drugs used for sepsis (rAPC and rNAPc2) were the first effective post-exposure therapies for Ebola virus infection with $17 \%$ - 33\% survival but administration required continuous intravenous infusion [2,3], which is difficult to achieve during patient management in Central African health care settings. The vesicular stomatitis virus-based filovirus vaccines resulted in an unprecedented $50 \%$ protection against Ebola virus challenge [4] and $100 \%$ against Marburg virus challenge [5] in rhesus macaques when administered $\sim 30$ minutes post-infection and showed effectiveness (33\% survival) against Marburg virus as late as $48 \mathrm{~h}$. Unfortunately, these attenuated live vaccines continue to face a myriad of regulatory hurdles due to remaining safety concerns. Recently, modulatory RNA treatments have been pursued with success. A cocktail of 3 different siRNAs (against L, VP24 and VP35) encapsulated in stable nucleic acid-lipid particles (SNALPs) provided up to $100 \%$ protection against Ebola virus when given 30 min post-exposure [6], while positively charged phosphorodiamidate morpholino oligomers protected $62.5 \%$ of rhesus macaques against Ebola virus and 100\% of cynomolgus macaques from Marburg virus disease when given within 30 min of infection [7]. In these cases, the significant cost of the large quantity of synthetic RNA required for protection in humans remains a substantial barrier.

Antibody-mediated therapeutics, once thought impossible, have taken the lead in terms of survival and delayed treatment. Purified IgG from vaccinated and subsequently challenged nonhuman primate survivors provided $100 \%$ protection from Ebola or Marburg virus challenge when administered up to $48 \mathrm{~h}$ post-infection [8]. This advance was followed closely by a cocktail of 3 monoclonal antibodies that was $100 \%$ effective at $24 \mathrm{~h}$ and $50 \%$ effective at 48 $\mathrm{h}$ in preventing death against Ebola virus challenge in cynomolgus macaques [9]. Later, this same cocktail when combined with Adenovirus-vectored interferon- $\alpha$ was $75 \%$ and $100 \%$ effective in cynomolgus and rhesus macaques, respectively, when administered $72 \mathrm{~h}$ postinfection. Similarly, an alternate cocktail of 3 humanized monoclonal antibodies (MB-003) provided 100\% (1 h) and 67\% protection ( $24 \mathrm{~h}$ and $48 \mathrm{~h}$ post-infection) [10] in the Ebola virus rhesus macaque model and $43 \%$ survival when treatment occurred at $120 \mathrm{~h}$ post-infection following onset of viremia and fever [11]. These two trials represent the first true therapeutic interventions whereby in response to a diagnostic indication, the onset of viremia and/or fever, treatment is initiated.

Until further experiments are performed with BCX4430, the breadth of protection against the multiple species of Ebola and Marburg viruses remains to be evaluated in nonhuman primate models and the maximum effective delay in treatment needs to be determined (and will likely vary by challenge virus). In Marburg virus (strain RAVN)-infected mice, treatment could be delayed up to $96 \mathrm{~h}$ post-infection with comparable survival to earlier treatment regimens, while in guinea pigs treatment could be 


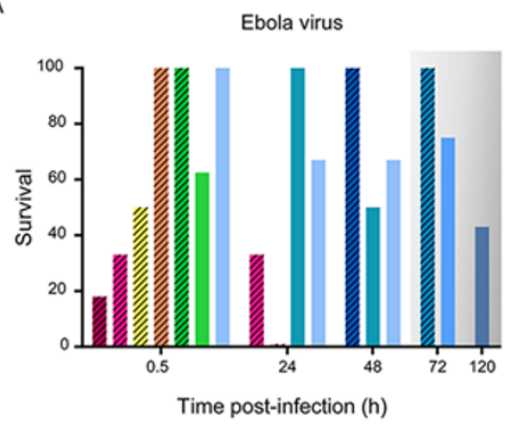

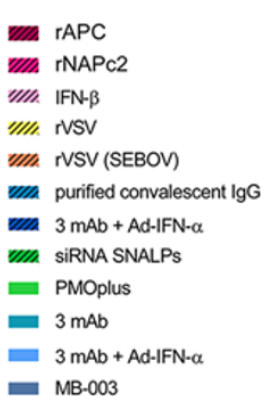

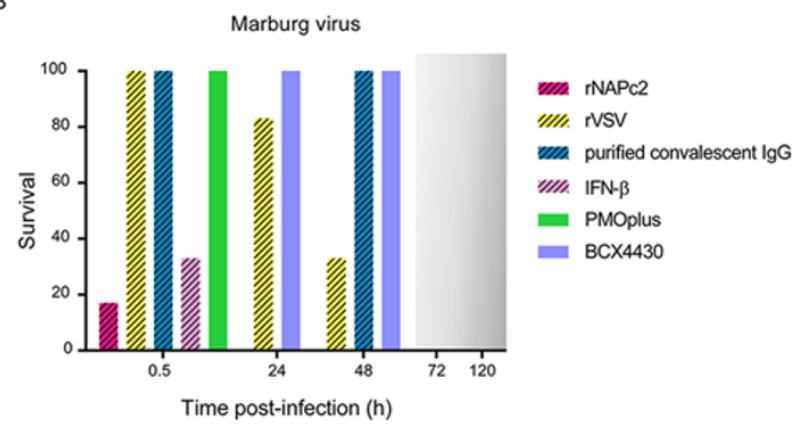

Figure 1 Survival in the rhesus macaque (diagonal lined) and cynomolgus macaque (solid) models of Ebola (A) and Marburg (B) virus infection following post-exposure intervention strategies. The diagnostic window when detection of viremia and/or onset of fever occurs is indicated in shading. Recombinant activated protein C (rAPC), recombinant nematode anticoagulant protein c2 (rNAPc2), recombinant vesicular stomatitis virus (rVSV), Sudan ebolavirus (SEBOV) challenge, small interfering (si)RNA stable nucleic acid lipid particles (SNALPs), monoclonal antibody (mAb), Adenovirus-vectored (Ad) interferon (IFN)- $\alpha$, phosphorodiamidate oligonucleotides (PMO), cocktail of $3 \mathrm{mAb}$ (MB-003).

delayed up to $48 \mathrm{~h}$ (intraperitoneal challenge) or $72 \mathrm{~h}$ (aerosol challenge). This suggests that the compound may retain some effectiveness when treatment starts in the time frame of diagnostic confirmation. Unfortunately, the current treatment window does not overlap with the onset of viremia, making treatment necessary prior to laboratory confirmation. However, if BCX 4430 has minimal side effects and is cost-effective, it could be widely administered to high-risk individuals in an outbreak setting. The available data suggests that oral delivery may be possible but kinetics may be more favorable following intramuscular injection - both being conducive routes of delivery in outbreak regions, but multiple applications per day may be more difficult to achieve in infrastructure-poor locations. In general, the time point of detectable viremia and the therapeutic window are beginning to overlap for multiple approaches, providing the first opportunities for targeted therapeutic intervention. Until now all other interventions have been difficult to implement, lack approval or are of limited practical use in outbreak settings.

Currently no prophylactic or therapeutic vaccines are approved. Monoclonal antibody combinations are costly to produce and are not currently available in mass quantities. Limited quantities of convalescent plasma also prohibit widespread use at present. In addition, all of these approaches also suffer from being virus species specific. Similar limitations are associated with modulatory RNA treatment approaches. The attraction of a small molecule inhibitor with broad spectrum filovirus efficacy is obvious as production and safety trials can follow traditional approaches and could then be implemented during an outbreak. If BCX4430 has a good safety profile, assessment in a phase I clinical trial should be straightforward potentially leading to relatively fast investigational new drug (IND) approval, with subsequent prophylactic use in a larger population during an outbreak seeming possible. Combination therapy with one of the other promising approaches could be beneficial and should also be considered. For laboratory workers as well as military and medical personnel this may also be an attractive intervention following an exposure, as we urgently wait for approval of one of the numerous prophylactic vaccines. Unfortunately, and despite much attention over the past decade, there is once again nothing to offer to those affected by the current Ebola virus outbreak in Guinea [12]. Unless promising treatments are pursued to some end, there will still be no intervention when the next outbreak strikes.

\section{Darryl Falzarano ${ }^{1}$,} Heinz Feldmann ${ }^{1}$

${ }^{1}$ Laboratory of Virology, Division of Intramural Research, National Institute of Allergy and Infectious Diseases, National Institutes of Health, Hamilton, MT 59840, USA

Correspondence: Heinz Feldmann

E-mail: feldmannh@niaid.nih.gov

\section{References}

1 Warren TK, Wells J, Panchal RG, et al. Nature 2014; 508:402-405

2 Hensley LE, Stevens EL, Yan SB, et al. $J$ Infect Dis 2007; 196 Suppl 2:S390-S399.

3 Geisbert TW, Hensley LE, Jahrling PB, et al. Lancet 2003; 362:1953-1958.

4 Feldmann H, Jones SM, Daddario-DiCaprio KM, et al. PLoS Pathog 2007; 3:e2.

5 Daddario-DiCaprio KM, Geisbert TW, Ströher $\mathrm{U}$, et al. Lancet 2006; 367:1399-1404.

6 Geisbert TW, Lee AC, Robbins M, et al. Lancet 2010; 375:1896-1905.

7 Warren TK, Warfield KL, Wells J, et al. Nat Med 2010; 16:991-994.

8 Dye JM, Herbert AS, Kuehne AI, et al. Proc Natl Acad Sci USA 2012; 109:5034-5039.

9 Qiu X, Audet J, Wong G, et al. Sci Transl Med 2012; 4:138ra181.

10 Olinger GG Jr, Pettitt J, Kim D, et al. Proc Natl Acad Sci USA 2012; 109:18030-18035.

11 Pettitt J, Zeitlin L, Kim do H, et al. Sci Transl Med 2013; 5:199ra113.

12 ProMED-mail (2014) Ebola Virus Disease Guinea (03): WHO Update, Travel Health Advisories. 20140326.2359361 (Accessed $3 / 28 / 2014$ ). 